\title{
Hematology, plasma biochemistry, and hormonal analysis of captive Louisiana pine snakes (Pituophis ruthveni): effects of intrinsic factors and analytical methodology
}

\author{
Luca Giori $^{1}$ (I) Nicole I. Stacy ${ }^{2} \cdot$ Michael Ogle $^{3} \cdot$ Stephen Nelson $^{3} \cdot$ Kellie A. Fecteau $^{1} \cdot$ Andrew C. Cushing $^{4}$
}

Received: 7 March 2019 / Accepted: 17 July 2019 / Published online: 9 August 2019

(C) The Author(s) 2019

\begin{abstract}
Blood analyte data are useful in health assessments and management of reptiles. There is a knowledge gap for blood analyte data of the endangered Louisiana pine snake (LPS; Pituophis ruthveni). The objectives of this study were to provide baseline hematology, plasma biochemical, and hormone data of captive LPS, to compare the data in juvenile and adult snakes and in adult snakes by sex, and to investigate methodological differences in hormone (serum vs. plasma) and protein analyses (total solids versus total protein). Blood samples from apparently healthy captive LPS were analyzed for hematology and plasma biochemistry $(n=11)$ and plasma and serum hormone analyses $(n=9)$. Packed cell volume $(\mathrm{PCV})$ and absolute heterophils were significantly higher in adult compared with juvenile LPS, while PCV, white blood cell count, and absolute lymphocytes were higher in adult males compared with adult females. Significantly higher plasma concentrations were found in adults compared with juveniles for calcium, total protein, total solids, albumin, globulins, and bile acids. No significant differences were observed in $17 \beta$-estradiol measured in serum and plasma when comparing adults and juveniles and for $17 \beta$-estradiol in adult males and females. Plasma concentrations of $17 \beta$-estradiol were significantly lower than in serum. Serum testosterone in two adult males was 8.33 and $35.53 \mathrm{nmol} / \mathrm{L}$, respectively, while it was undetectable in females and juveniles $(n=5)$. This study is the first to provide baseline information on blood analytes in endangered LPS, which will be useful for individual animals in managed care and as baseline for future population-level assessments.
\end{abstract}

Keywords Blood $\cdot$ Methodology $\cdot$ Plasma chemistry $\cdot$ Hormones $\cdot$ Reptile

Electronic supplementary material The online version of this article (https://doi.org/10.1007/s00580-019-03030-w) contains supplementary material, which is available to authorized users.

Luca Giori

lgiori@utk.edu

1 Department of Biomedical and Diagnostic Sciences, College of Veterinary Medicine, University of Tennessee, 2407 River Drive, Knoxville, TN 37996, USA

2 Department of Comparative, Diagnostic, and Population Medicine, College of Veterinary Medicine, University of Florida, 2015 SW 16th Ave, Gainesville, FL 32608, USA

3 Zoo Knoxville, 3500 Knoxville Zoo Drive, Knoxville, TN 37914 , USA

4 Department of Small Animal Clinical Sciences, University of Tennessee, 2407 River Drive, Knoxville, TN 37996, USA

\section{Introduction}

Pituophis is a genus of non-venomous large-bodied constrictor snakes belonging to the Colubridae family, which is considered the largest and most structurally diverse snake group, including about $50 \%$ of all known living snake species (Uetz et al. 2018; Vitt and Caldwell 2014). Previously endemic to western Louisiana and eastern Texas, the Louisiana pine snake (LPS; Pituophis ruthveni) population has declined precipitously in recent decades and is now restricted to highly fragmented and isolated patches of both southeastern states after degradation of its natural habitat, mostly due to intensive silviculture, and is thus categorized as endangered by the IUCN Red List (International Union for Conservation of Nature 2019; Rudolph et al. 2006).

Hematological and biochemical analytes are essential baseline diagnostics in the health assessment of any species. There is extensive variation in such data between species and even genus, and published reference data are lacking for many 
snake species. In addition, significant variation can occur within a given species due to intrinsic and extrinsic factors such as ambient environment, season, analytical methodology, physiological state, and diet (Campbell 2015c; Stacy et al. 2011). Hormone analysis in snakes is not routinely performed due to lack of readily validated assays for diagnostic use and thus most frequently used in research studies offering insights into reproductive physiology, breeding suitability, or timing and may offer an alternative tool for sexing as demonstrated in previous studies (Edwards and Jones 2001; Jones 2011; Taylor and DeNardo 2011; Taylor et al. 2004).

Published literature about LPS is primarily focused on biological investigations on natural habitat, growth rates, and ecological behaviors of the species (Ealy et al. 2004; Himes 2001; Himes et al. 2002; Pierce et al. 2014; Rudolph et al. 2007). To the authors' knowledge, information on hematology, plasma biochemistry, and hormone data in this species is unavailable to date. Blood analyte data can provide useful information for answering biological and ecological questions regarding an individual and even a population if baseline data with comparable methodology is available for reference. The objectives of this study were to (1) provide baseline hematological, plasma biochemical, and hormone data of captive LPS, to (2) compare the data in juvenile and adult snakes and in adult snakes by sex, and to (3) investigate methodological differences in hormone (serum vs. plasma) and protein analyses (total solids versus total protein).

\section{Materials and methods}

Eleven captive LPS housed at Zoo Knoxville, TN, USA, were included in this study. The apparently healthy snakes included five juveniles ( $<18$ months) and six adults (8-12 years). Juvenile and adult weights ranged from 275 to $440 \mathrm{~g}$ and from 1490 to $4200 \mathrm{~g}$, respectively. Snout-vent length (SVL) and total length (TL) varied from 87 to $101.5 \mathrm{~cm}$ and from 97 to $114 \mathrm{~cm}$ for the juveniles and from 136 to $198 \mathrm{~cm}$ and from 158 to $230 \mathrm{~cm}$ for adults.

The snakes were housed in a rack system and continuously maintained at $25-30{ }^{\circ} \mathrm{C}$. The adults were provided with a $34{ }^{\circ} \mathrm{C}$ basking spot from 1st March to 15th December each year. From mid-December to the end of February, adults are in brumation and the temperature was maintained between 10 and $13{ }^{\circ} \mathrm{C}$ to mirror natural habitat conditions. Juveniles were fed weekly with an appropriately sized rodent. The adults were fed two to three small or medium rats in the spring for the upcoming breeding season and then one to two small or medium rats weekly throughout the rest of the active season. For all snakes, the length of the artificial daylight is $9 \mathrm{~h}$ yearround. The snakes were sampled as part of routine examinations on the same day in fall, which is outside the known breeding season for this species. Snakes were transported from their tubs to the zoo clinic in individual cotton bags, were weighed, and a physical examination was performed by one of the authors (AC). All snakes were physically examined and found to be apparently healthy (e.g., normal feeding behavior, absence of external injuries or lesions) and were not on any medications for at least 4 weeks prior to sampling. Total length (TL) was also recorded. The sex of each snake had previously been assessed by probing.

\section{Blood collection}

Blood was collected by venipuncture of the ventral coccygeal vein using a 22-gauge needle attached to a $3-\mathrm{mL}$ syringe. In order to facilitate blood collection from the tail, the snakes were restrained with the head elevated above the level of the tail. For two smaller snakes, a cardiac puncture was elected in order to obtain the minimum amount of blood to perform the analysis. Immediately after collection, blood was aliquoted into three different tubes: one heparinized tube without gel separator (BD Microtainer® lithium heparin tube, Franklin Lakes, NJ, USA) used for hematological evaluation, one heparinized tube with gel separator (BD Microtainer® ${ }^{\circledR}$ PST $^{\mathrm{TM}}$ lithium heparin tube, Franklin Lakes, NJ, USA) used for plasma biochemistry, and one spray-coated silica tube for serum (BD Vacutainer® Plus plastic serum tube, Franklin Lakes, NJ, USA) used for hormone analysis. Two blood films were immediately prepared by author LG from each heparinized blood sample and allowed to air dry.

\section{Hematological and plasma biochemistry analysis}

Blood samples were processed and analyzed at the University of Tennessee College of Veterinary Medicine Clinical Pathology Laboratory (UTCVM-CPL) within $3 \mathrm{~h}$ of collection for routine hematology and plasma biochemical analysis. In addition, an equal volume of $25 \mu \mathrm{L}$ of whole blood and supravital dye New Methylene Blue (NMB) were mixed and incubated for $15 \mathrm{~min}$ for reticulocyte counts (No. reticulocytes/1000 red blood cell (RBC)) that were performed by one author (LG) following the standard protocol used at the UTCVM-CPL. For routine hematological analysis, heparinized blood was spun at $16,128 \mathrm{~g}$ for $10 \mathrm{~min}$ (PowerSpin $\mathrm{MH}$ Centrifuge, Unico, Dayton, NJ) in capillary tubes to obtain the spun packed cell volume (PCV). A white blood cell (WBC) count was performed by an experienced technologist using the semidirect technique using phloxine B (Avian Leukopet, Vetlab Supply, Palmetto Bay, FL, USA) as previously described (Campbell 2015b; Geffre et al. 2009b; Rush et al. 2016). Both blood films were stained with Wright-Giemsa stain with an automatic methanolic slide stainer (Mod.7151, Wescor, Inc., Logan, UT). The same technologist performed WBC differential, WBC morphology, RBC morphology, thrombocyte estimate, and hemoparasite review on blood 
smears. Leukocytes were classified as heterophils, lymphocytes, monocytes, eosinophils, basophils, and azurophils. Thrombocytes were assessed as "adequate" or "not adequate" based on the size and number of thrombocyte clumps. Blood cell sizes were evaluated using an eyepiece graticule with a scale previously calibrated by a stage micrometer.

After centrifugation of the heparinized blood at $700 \times \mathrm{g} \mathrm{rpm}$ for $10 \mathrm{~min}$ (Sero-Fuge centrifuge, Becton, Dickinson and Co, Franklin Lakes, NJ), plasma was separated and transferred in specific microcups for biochemistry analysis with a Cobas c501 analyzer (Roche Diagnostics, Indianapolis IN, USA). Total solids (TS) were assessed using a refractometer (Mod.1310400A, Reichert Analytical Instruments, Depew, NY). The UTCVM-CPL biochemistry panel for reptiles included albumin (ALB), alkaline phosphates (ALKP), aspartate aminotransaminase (AST), bile acids (BA), total calcium (CA), creatine kinase (CK), cholesterol (CHOL), globulins (GLOB), glucose (GLU), lactate dehydrogenase (LDH), phosphorus $(\mathrm{P})$, urea $(\mathrm{BUN})$, total protein $(\mathrm{TP})$, and uric acid (UA).

\section{Hormonal analysis}

An adequate amount of plasma in addition to serum obtained after centrifugation of non-heparinized blood (at $700 \mathrm{~g}$ for $12 \mathrm{~min}$, Thermo Scientific IEC CL10 centrifuge, Thermofisher Scientific, Waltham, MA) was aliquoted, kept at $4{ }^{\circ} \mathrm{C}$, and transported to the UTCVM Diagnostic Endocrinology Service within $30 \mathrm{~min}$ for analysis of the following hormones: $17 \beta$-estradiol, 17-hydroxyprogesterone (17OHP), progesterone, and testosterone. $17 \beta$-estradiol and $17 \mathrm{OHP}$ were measured in duplicate both in serum and plasma samples for 8 of the 11 snakes using radioimmunoassay (17 $\beta$ estradiol Radioimmunoassay, MP Biomedicals, USA; $17-$ hydroxyprogesterone Radioimmunoassay, MP Biomedicals, USA) (Tumkiratiwong et al. 2012). Progesterone and testosterone were measured in duplicate both in serum and plasma samples for 8 of the 11 snakes using a chemiluminescence assay (IMMULITE® 2000XPi Immunoassay Analyzer, Siemens Healthcare, USA).

Validation of the chemiluminescence and radioimmunoassays was performed by determining inter- and intra-assay precision and accuracy as suggested by the American Society of Veterinary Clinical Pathology (ASVCP) Quality Control Guidelines and Lee et al. (2006). Intra- and inter-assay coefficients of variation for $17 \beta$-estradiol and progesterone assays were $4.4 \%$ and $5.8 \%$, and $5.8 \%$ and $8.2 \%$, respectively.

Assays were validated 3 months prior to this study, using pooled serum obtained from fresh blood samples collected from the same six LPS adults (three females and three males) in a pilot experiment. Recovery of a known amount of progesterone assay control material (Bio-Rad Lyphochek; immunoassay Plus control) added to snake serum was
$104 \%$, while recovery of a known amount of $17 \beta$-estradiol assay standard added to snake serum was $92 \%$.

The limit of detection for $17 \beta$-estradiol, progesterone, and testosterone were $<36.71 \mathrm{pmol} / \mathrm{L},<0.64 \mathrm{nmol} / \mathrm{L}$, and $<$ $0.12 \mathrm{nmol} / \mathrm{L}$, respectively.

\section{Statistical analysis}

Biochemistry, hematology, and hormone data were analyzed using the non-parametric Kruskal-Wallis test to detect the effects of gender in adults and age (i.e., adult vs. juvenile). The presence of a single male in the juvenile group precluded analysis by sex in this age group. The difference in $17 \beta$ estradiol concentrations between serum and plasma was analyzed using paired $t$ test for repeated measures. Significance was identified at $p<0.05$. All analyses were conducted in SAS9.4 for Windows $64 \times$ (SAS Institute, Cary, NC). The correlation between total solids by refractometer and total protein concentration measured by the chemistry analyzer (biuret method) was calculated with the Spearman rank correlation method because data were not normally distributed. Correlation results were categorized as very high $(r>0.90)$, high ( $r=0.70$ to 0.90$)$, moderate $(r=0.50$ to 0.70 ), or low $(r=0.30$ to 0.50 ) (Mukaka 2012). Histograms for all hematology and chemistry analytes are provided in the Electronic supplementary materials. Data on 17-hydroxyprogesterone were excluded from the study due to all samples resulting below the limit of detection of the assay $(0.03 \mathrm{nmol} / \mathrm{L})$, presumptively due to lack of sufficient cross-reactivity of the assay. Statistical analysis was not performed on testosterone due to female $(n=2)$ and juvenile $(n=3)$ data resulting below the detection limit of the assay $(<0.12 \mathrm{nmol} / \mathrm{L})$, but two male samples had detectable concentrations. Due to low sample number, we only compared estrogen concentrations from serum and heparinized plasma statistically.

\section{Results}

Results of hematological analyses are shown in Tables 1 and 2. All samples had minimal trace values of lipemia, hemolysis, and "icterus" lower than 30 (on a scale of 500 by chemistry analyzer) except for one sample in an adult male with mild hemolysis $(114 / 500)$ which was not considered to affect chemistry analyte data. Additional visible evaluation did not show any overt plasma discolorations.

Packed cell volume was significantly higher in adults compared with juveniles $(p=0.0274)$ and in adult males compared with adult females $(p=0.0431)$. Lymphocytes were the predominant WBC type observed in all snakes, followed by heterophils. Heterophils were significantly higher in adults compared with juveniles $(p=0.0441)$. Monocytes and azurophils 
Table 1 Descriptive hematological and plasma biochemical data in adult $(n=6)$ and juvenile $(n=5)$ apparently healthy captive Louisiana pine snakes (Pituophis ruthveni)

\begin{tabular}{|c|c|c|c|c|c|c|c|}
\hline Analyte & Life stage & Mean & $\mathrm{SD}$ & Median & Min & Max & \\
\hline PCV (L/L) & $\begin{array}{l}\text { Adult } \\
\text { Juvenile }\end{array}$ & $\begin{array}{l}0.38 \\
0.26\end{array}$ & $\begin{array}{l} \pm 0.5 \\
\pm 0.7\end{array}$ & $\begin{array}{l}0.385 \\
0.30\end{array}$ & $\begin{array}{l}0.29 \\
0.17\end{array}$ & $\begin{array}{l}0.43 \\
0.34\end{array}$ & $*$ \\
\hline WBC $\left(\times 10^{9} / \mathrm{L}\right)$ & $\begin{array}{l}\text { Adult } \\
\text { Juvenile }\end{array}$ & $\begin{array}{l}13.15 \\
6.62\end{array}$ & $\begin{array}{l} \pm 6.01 \\
\pm 1.33\end{array}$ & $\begin{array}{l}15.75 \\
7.20\end{array}$ & $\begin{array}{l}4.90 \\
4.50\end{array}$ & $\begin{array}{l}20.7 \\
8.30\end{array}$ & \\
\hline Heterophils $\left(\times 10^{9} / \mathrm{L}\right)$ & $\begin{array}{l}\text { Adult } \\
\text { Juvenile }\end{array}$ & $\begin{array}{l}1.74 \\
0.74\end{array}$ & $\begin{array}{l} \pm 0.68 \\
\pm 0.11\end{array}$ & $\begin{array}{l}1.82 \\
0.70\end{array}$ & $\begin{array}{l}0.60 \\
0.58\end{array}$ & $\begin{array}{l}2.69 \\
0.90\end{array}$ & $*$ \\
\hline Lymphocytes $\left(\times 10^{9} / \mathrm{L}\right)$ & $\begin{array}{l}\text { Adult } \\
\text { Juvenile }\end{array}$ & $\begin{array}{l}10.20 \\
4.57\end{array}$ & $\begin{array}{l} \pm 4.98 \\
\pm 0.89\end{array}$ & $\begin{array}{l}12.0 \\
4.80\end{array}$ & $\begin{array}{l}3.04 \\
3.10\end{array}$ & $\begin{array}{l}16.97 \\
5.55\end{array}$ & \\
\hline Monocytes $\left(\times 10^{9} / \mathrm{L}\right)$ & $\begin{array}{l}\text { Adult } \\
\text { Juvenile }\end{array}$ & $\begin{array}{l}0.50 \\
0.46\end{array}$ & $\begin{array}{l} \pm 0.36 \\
\pm 0.21\end{array}$ & $\begin{array}{l}0.46 \\
0.40\end{array}$ & $\begin{array}{l}0.10 \\
0.20\end{array}$ & $\begin{array}{l}1.07 \\
0.80\end{array}$ & \\
\hline Azurophils $\left(\times 10^{9} / \mathrm{L}\right)$ & $\begin{array}{l}\text { Adult } \\
\text { Juvenile }\end{array}$ & $\begin{array}{l}0.46 \\
0.66\end{array}$ & $\begin{array}{l} \pm 0.44 \\
\pm 0.21\end{array}$ & $\begin{array}{l}0.37 \\
0.58\end{array}$ & $\begin{array}{l}0.0 \\
0.40\end{array}$ & $\begin{array}{l}1.38 \\
0.90\end{array}$ & \\
\hline Basophils $\left(\times 10^{9} / \mathrm{L}\right)$ & $\begin{array}{l}\text { Adult } \\
\text { Juvenile }\end{array}$ & $\begin{array}{l}0.07 \\
0.30\end{array}$ & $\begin{array}{l} \pm 0.09 \\
\pm 0.33\end{array}$ & $\begin{array}{l}0.0 \\
0.20\end{array}$ & $\begin{array}{l}0.0 \\
0.0\end{array}$ & $\begin{array}{l}0.20 \\
0.90\end{array}$ & \\
\hline Eosinophils $\left(\times 10^{9} / \mathrm{L}\right)$ & $\begin{array}{l}\text { Adult } \\
\text { Juvenile }\end{array}$ & $\begin{array}{l}0.0 \\
0.0\end{array}$ & $\begin{array}{l} \pm 0.0 \\
\pm 0.0\end{array}$ & $\begin{array}{l}0.0 \\
0.0\end{array}$ & $\begin{array}{l}0.0 \\
0.0\end{array}$ & $\begin{array}{l}0.0 \\
0.0\end{array}$ & \\
\hline ALKP (nkat/L) & $\begin{array}{l}\text { Adult } \\
\text { Juvenile }\end{array}$ & $\begin{array}{l}767 \\
517\end{array}$ & $\begin{array}{l} \pm 483 \\
\pm 100\end{array}$ & $\begin{array}{l}600 \\
483\end{array}$ & $\begin{array}{l}283 \\
400\end{array}$ & $\begin{array}{l}1434 \\
650\end{array}$ & \\
\hline AST (nkat/L) & $\begin{array}{l}\text { Adult } \\
\text { Juvenile }\end{array}$ & $\begin{array}{l}233 \\
83\end{array}$ & $\begin{array}{l} \pm 67 \\
\pm 350\end{array}$ & $\begin{array}{l}200 \\
83\end{array}$ & $\begin{array}{l}167 \\
33\end{array}$ & $\begin{array}{l}350 \\
933\end{array}$ & \\
\hline LDH (nkat/L) & $\begin{array}{l}\text { Adult } \\
\text { Juvenile }\end{array}$ & $\begin{array}{l}1584 \\
1300\end{array}$ & $\begin{array}{l} \pm 800 \\
\pm 1367\end{array}$ & $\begin{array}{l}1300 \\
600\end{array}$ & $\begin{array}{l}733 \\
167\end{array}$ & $\begin{array}{l}2917 \\
3934\end{array}$ & \\
\hline Creatine kinase (nkat/L) & $\begin{array}{l}\text { Adult } \\
\text { Juvenile }\end{array}$ & $\begin{array}{l}7701 \\
10,219\end{array}$ & $\begin{array}{l} \pm 2751 \\
\pm 8135\end{array}$ & $\begin{array}{l}6801 \\
6935\end{array}$ & $\begin{array}{l}4701 \\
1750\end{array}$ & $\begin{array}{l}11,919 \\
20,137\end{array}$ & \\
\hline Phosphorus (mmol/L) & $\begin{array}{l}\text { Adult } \\
\text { Juvenile }\end{array}$ & $\begin{array}{l}1.4 \\
1.3\end{array}$ & $\begin{array}{l} \pm 0.3 \\
\pm 0.3\end{array}$ & $\begin{array}{l}1.3 \\
1.1\end{array}$ & $\begin{array}{l}1.1 \\
1.0\end{array}$ & $\begin{array}{l}1.7 \\
1.8\end{array}$ & \\
\hline Calcium (mmol/L) & $\begin{array}{l}\text { Adult } \\
\text { Juvenile }\end{array}$ & $\begin{array}{l}4.4 \\
3.7\end{array}$ & $\begin{array}{l} \pm 0.2 \\
\pm 0.4\end{array}$ & $\begin{array}{l}4.4 \\
3.5\end{array}$ & $\begin{array}{l}3.9 \\
3.5\end{array}$ & $\begin{array}{l}4.7 \\
4.5\end{array}$ & $*$ \\
\hline $\mathrm{Ca}: \mathrm{P}$ ratio & $\begin{array}{l}\text { Adult } \\
\text { Juvenile }\end{array}$ & $\begin{array}{l}3.3 \\
3.1\end{array}$ & $\begin{array}{l} \pm 0.6 \\
\pm 0.4\end{array}$ & $\begin{array}{l}3.3 \\
3.2\end{array}$ & $\begin{array}{l}2.5 \\
2.5\end{array}$ & $\begin{array}{l}4.1 \\
3.7\end{array}$ & \\
\hline Glucose $(\mathrm{mmol} / \mathrm{L})$ & $\begin{array}{l}\text { Adult } \\
\text { Juvenile }\end{array}$ & $\begin{array}{l}4.1 \\
3.4\end{array}$ & $\begin{array}{l} \pm 1.4 \\
\pm 0.9\end{array}$ & $\begin{array}{l}3.9 \\
3.1\end{array}$ & $\begin{array}{l}2.4 \\
2.8\end{array}$ & $\begin{array}{l}6.8 \\
5.2\end{array}$ & \\
\hline Total Protein $(\mathrm{g} / \mathrm{L})$ & $\begin{array}{l}\text { Adult } \\
\text { Juvenile }\end{array}$ & $\begin{array}{l}71.0 \\
53.0\end{array}$ & $\begin{array}{l} \pm 4.0 \\
\pm 5.0\end{array}$ & $\begin{array}{l}70.0 \\
51.0\end{array}$ & $\begin{array}{l}65.0 \\
48.0\end{array}$ & $\begin{array}{l}77.0 \\
63.0\end{array}$ & $*$ \\
\hline Total Solids (g/L) & $\begin{array}{l}\text { Adult } \\
\text { Juvenile }\end{array}$ & $\begin{array}{l}97.0 \\
66.0\end{array}$ & $\begin{array}{l} \pm 7.0 \\
\pm 6.0\end{array}$ & $\begin{array}{l}98.0 \\
65.0\end{array}$ & $\begin{array}{l}84.0 \\
57.0\end{array}$ & $\begin{array}{l}105.0 \\
76.0\end{array}$ & $*$ \\
\hline Albumin $(\mathrm{g} / \mathrm{L})$ & $\begin{array}{l}\text { Adult } \\
\text { Juvenile }\end{array}$ & $\begin{array}{l}20.0 \\
17.0\end{array}$ & $\begin{array}{l} \pm 1.2 \\
\pm 1.4\end{array}$ & $\begin{array}{l}20.0 \\
18.0\end{array}$ & $\begin{array}{l}18.0 \\
15.0\end{array}$ & $\begin{array}{l}22.0 \\
19.0\end{array}$ & $*$ \\
\hline Globulin (g/L) & $\begin{array}{l}\text { Adult } \\
\text { Juvenile }\end{array}$ & $\begin{array}{l}51.0 \\
36.0\end{array}$ & $\begin{array}{l} \pm 3.4 \\
\pm 4.4\end{array}$ & $\begin{array}{l}50.0 \\
33.0\end{array}$ & $\begin{array}{l}47.0 \\
32.0\end{array}$ & $\begin{array}{l}58.0 \\
44.0\end{array}$ & $*$ \\
\hline A:G ratio & $\begin{array}{l}\text { Adult } \\
\text { Juvenile }\end{array}$ & $\begin{array}{l}0.4 \\
0.5\end{array}$ & $\begin{array}{l} \pm 0.0 \\
\pm 0.1\end{array}$ & $\begin{array}{l}0.4 \\
0.5\end{array}$ & $\begin{array}{l}0.3 \\
0.4\end{array}$ & $\begin{array}{l}0.4 \\
0.6\end{array}$ & $*$ \\
\hline Cholesterol (mmol/L) & $\begin{array}{l}\text { Adult } \\
\text { Juvenile }\end{array}$ & $\begin{array}{l}18.6 \\
12.6\end{array}$ & $\begin{array}{l} \pm 2.7 \\
\pm 4.9\end{array}$ & $\begin{array}{l}18.6 \\
11.0\end{array}$ & $\begin{array}{l}14.4 \\
9.2\end{array}$ & $\begin{array}{l}23.3 \\
22.3\end{array}$ & \\
\hline Bile Acids (mmol/L) & $\begin{array}{l}\text { Adult } \\
\text { Juvenile }\end{array}$ & $\begin{array}{l}9.7 \\
3\end{array}$ & $\begin{array}{l} \pm 4.4 \\
\pm 2.1\end{array}$ & $\begin{array}{l}10 \\
2\end{array}$ & $\begin{array}{l}3 \\
1\end{array}$ & $\begin{array}{l}15 \\
7\end{array}$ & $*$ \\
\hline Uric acid $(\mathrm{mmol} / \mathrm{L})$ & $\begin{array}{l}\text { Adult } \\
\text { Juvenile }\end{array}$ & $\begin{array}{l}547.2 \\
267.7\end{array}$ & $\begin{array}{l} \pm 315.2 \\
\pm 113.0\end{array}$ & $\begin{array}{l}463.9 \\
208.2\end{array}$ & $\begin{array}{l}267.7 \\
154.6\end{array}$ & $\begin{array}{l}1171.8 \\
475.8\end{array}$ & \\
\hline
\end{tabular}

A: G ratio, plasma albumin-to-globulin ratio; Ca:P ratio, calcium-to-phosphorus ratio; $A L K P$, alkaline phosphatase; $A S T$, aspartate aminotransferase; $L D H$, lactate dehydrogenase

${ }^{*} p<0.05$, statistical significance 
were similar in all samples. No significant differences were observed between adults and juveniles for WBC counts $(p=$ $0.2012)$, lymphocytes $(p=0.2012)$, monocytes $(P=1.0000)$, azurophils $(p=0.1190)$, and basophils $(p=0.1944)$. WBC counts $(p=0.0495)$, and lymphocytes $(p=0.0495)$ were significantly higher in adult males versus adult females.

The morphology of RBC was consistent with descriptions in other snakes (Campbell 2015c; Jenkins-Perez 2008; Stacy et al. 2011): mature erythrocytes were ellipsoid and nucleated (6-8 $\mu \mathrm{m}$ wide, 20-22 $\mu \mathrm{m}$ long; Fig. 1a-1) containing orangepink homogeneous cytoplasm. Single small punctate basophilic inclusions most consistent with previously described membranes of degenerate organelles were occasionally present (Campbell 2015c). Nuclei were centrally located, with irregular nuclear margins (from oval to trapezoid) and dark blue densely clumped chromatin. Immature erythrocytes (polychromatophils by Wright-Giemsa; reticulocytes by NMB) were observed in adult and juvenile individuals (mean $5 \%$, range $2-10 \%$ using NMB). Higher numbers of polychromatophils, namely $8-10 \%$, including immature precursor stages and mitotic figures, were noticed in two juvenile individuals with lower PCV of 18 and 17\%, respectively, suggestive of an erythroid regenerative response (Fig. 1a, b, d). Since erythroid precursor stages can be misinterpreted as reactive or atypical lymphocytes, the morphology of these cells needs to be carefully evaluated. On NMB-stained blood films, residuals of RNA/cytoplasmic organelles were also observed in more mature $\mathrm{RBC}$ as multiple punctate darker blue precipitates (Fig. 1c) scattered throughout the entire cytoplasm. A clear abundant reticulum of precipitate surrounding the nuclei was apparent in earlier precursors.

Heterophils were the largest WBC (mean $21 \mu \mathrm{m}$; range 19$23 \mu \mathrm{m}$; Fig. 1e, f) and similar in morphology as described in other snake species (Stacy et al. 2011). Heterophil left-shifting or evidence of toxicity were absent in blood films from all snakes. Lymphocytes resembled those of mammalian and other reptile species. Lymphocytes ranged in size from 8 to $14 \mu \mathrm{m}$ (mean $11 \mu \mathrm{m}$, Fig. 1f). Larger reactive or plasmocytoid lymphocytes with deeply basophilic cytoplasm and pale perinuclear regions were rarely observed. Monocytes (Fig. 1j, k) and azurophils (Fig. 1g, h) were lower in number compared with heterophils and lymphocytes and varied in size from mean $16 \mu \mathrm{m}$ for monocytes and $18 \mu \mathrm{m}$ for azurophils. Neither melanomacrophages nor phagocytic macrophages were observed in any blood smears. Basophils were easily recognizable as small round cells (mean $8 \mu \mathrm{m}$; range 6 to $12 \mu \mathrm{m}$; Fig. 11) packed with numerous dark blue granules obscuring the nucleus. Occasionally, ruptured basophils were seen as naked nuclei surrounded by many scattered metachromatic granules. Eosinophils were not observed in any of the samples. Similar to other reptiles, thrombocytes in LPS had clear cytoplasm occasionally containing vacuoles and an ovoid/roundish nucleus with more densely clumped chromatin than lymphocytes. They were often clumped in blood smears due to activation during blood collection or due to heparin effects (Fig. 1i). Thrombocytes were considered adequate in blood films from all snakes. Hemoparasites were absent in all samples.

Results of plasma biochemical analyses are shown in Tables 1 and 2. Significantly higher concentrations were identified in adults compared with juveniles for the following analytes: CA $(p=0.0441)$; TP $(p=0.0061)$; TS $(p=0.0061)$; $\operatorname{ALB}(p=0.0253)$; GLOB $(p=0.0059)$; and BA $(p=0.0219)$. No statistical differences were observed for other biochemical analytes. The correlation between TS and TP was considered high $(r=0.82)$. No significant differences were identified in adult males and females.

Results of hormone analyses are shown in Table 3. Sufficient serum samples were obtained from four of six adult snakes and from three juveniles. Adequate amounts of plasma were available for four adults (for which serum was also available) and five juveniles. When concentrations of progesterone and testosterone were undetectable or below the limit of detection $(<0.64 \mathrm{nmol} / \mathrm{L}$ for progesterone and $<0.12 \mathrm{nmol} / \mathrm{L}$ for testosterone), data were excluded from the statistical analysis. For this reason, due to low sample numbers, statistical analyses for comparisons of testosterone and progesterone concentrations were not performed. No significant differences were observed in serum and plasma $17 \beta$-estradiol (serum, $p=$ 0.1573 ; plasma, $p=0.0864$ ) between adults and juveniles. No significant differences between adult males and females were seen in $17 \beta$-estradiol concentration in both samples (serum, $p=0.1213$; plasma, $p=0.1213$ ). A positive correlation $(r=0.821)$ was found between serum and plasma sample concentrations for $17 \beta$-estradiol, with serum concentrations trending consistently significantly higher $(p=0.0156)$ than those of plasma.

Although statistical analysis was not possible due to low sample number (e.g., insufficient sample volume or values below detection limit), an overt difference between testosterone concentrations was observed between adult males and females. The two adult males had serum (and plasma) testosterone of 35.53 (40.81) and $8.33 \mathrm{nmol} / \mathrm{L}$ (6.97), respectively, while the concentration of serum (and plasma) testosterone for two adult females was below the limit of detection of our assay $(<0.12 \mathrm{nmol} / \mathrm{L})$. Serum testosterone concentrations were below the limit of detection in three juvenile animals (one male, two females). Plasma testosterone concentrations were below detection limit in all juvenile animals (four females, one male).

\section{Discussion}

This report provides baseline hematology, chemistry, and hormone data in the endangered Louisiana pine snake, and documents evidence that age, sex, and analytical methodology 
Table 2 Descriptive hematological and plasma biochemical data in adult male $(n=3)$ and female $(n=3)$ apparently healthy captive Louisiana pine snakes (Pituophis ruthveni)

\begin{tabular}{|c|c|c|c|c|c|c|c|}
\hline Analyte & Sex & Mean & $\mathrm{SD}$ & Median & Min & Max & \\
\hline PCV (L/L) & $\begin{array}{l}\text { Male } \\
\text { Female }\end{array}$ & $\begin{array}{l}0.42 \\
0.34\end{array}$ & $\begin{array}{l} \pm 0.1 \\
\pm 0.4\end{array}$ & $\begin{array}{l}0.43 \\
0.37\end{array}$ & $\begin{array}{l}0.40 \\
0.29\end{array}$ & $\begin{array}{l}0.43 \\
0.37\end{array}$ & $*$ \\
\hline WBC $\left(\times 10^{9} / \mathrm{L}\right)$ & $\begin{array}{l}\text { Male } \\
\text { Female }\end{array}$ & $\begin{array}{l}17.87 \\
8.43\end{array}$ & $\begin{array}{l} \pm 2.01 \\
\pm 4.86\end{array}$ & $\begin{array}{l}16.7 \\
5.1\end{array}$ & $\begin{array}{l}16.20 \\
4.90\end{array}$ & $\begin{array}{l}20.70 \\
15.3\end{array}$ & * \\
\hline Heterophils $\left(\times 10^{9} / \mathrm{L}\right)$ & $\begin{array}{l}\text { Male } \\
\text { Female }\end{array}$ & $\begin{array}{l}2.26 \\
1.22\end{array}$ & $\begin{array}{l} \pm 0.36 \\
\pm 0.51\end{array}$ & $\begin{array}{l}2.3 \\
1.23\end{array}$ & $\begin{array}{l}1.80 \\
0.60\end{array}$ & $\begin{array}{l}2.69 \\
1.84\end{array}$ & \\
\hline Lymphocytes $\left(\times 10^{9} / \mathrm{L}\right)$ & $\begin{array}{l}\text { Male } \\
\text { Female }\end{array}$ & $\begin{array}{l}14.26 \\
6.15\end{array}$ & $\begin{array}{l} \pm 1.92 \\
\pm 3.61\end{array}$ & $\begin{array}{l}13 \\
4.20\end{array}$ & $\begin{array}{l}12.80 \\
3.04\end{array}$ & $\begin{array}{l}16.97 \\
11.20\end{array}$ & $*$ \\
\hline Monocytes $\left(\times 10^{9} / \mathrm{L}\right)$ & $\begin{array}{l}\text { Male } \\
\text { Female }\end{array}$ & $\begin{array}{l}0.57 \\
0.42\end{array}$ & $\begin{array}{l} \pm 0.21 \\
\pm 0.46\end{array}$ & $\begin{array}{l}0.10 \\
0.40\end{array}$ & $\begin{array}{l}0.10 \\
0.20\end{array}$ & $\begin{array}{l}1.07 \\
0.80\end{array}$ & \\
\hline Azurophils $\left(\times 10^{9} / \mathrm{L}\right)$ & $\begin{array}{l}\text { Male } \\
\text { Female }\end{array}$ & $\begin{array}{l}0.27 \\
0.64\end{array}$ & $\begin{array}{l} \pm 0.19 \\
\pm 0.53\end{array}$ & $\begin{array}{l}0.34 \\
0.58\end{array}$ & $\begin{array}{l}0.20 \\
0.40\end{array}$ & $\begin{array}{l}1.38 \\
0.90\end{array}$ & \\
\hline Basophils $\left(\times 10^{9} / \mathrm{L}\right)$ & $\begin{array}{l}\text { Male } \\
\text { Female }\end{array}$ & $\begin{array}{l}0.07 \\
0.07\end{array}$ & $\begin{array}{l} \pm 0.09 \\
\pm 0.09\end{array}$ & $\begin{array}{l}0.0 \\
0.0\end{array}$ & $\begin{array}{l}0.0 \\
0.0\end{array}$ & $\begin{array}{l}0.20 \\
0.20\end{array}$ & \\
\hline Eosinophils $\left(\times 10^{9} / \mathrm{L}\right)$ & $\begin{array}{l}\text { Male } \\
\text { Female }\end{array}$ & $\begin{array}{l}0.0 \\
0.0\end{array}$ & $\begin{array}{l} \pm 0.0 \\
\pm 0.0\end{array}$ & $\begin{array}{l}0.0 \\
0.0\end{array}$ & $\begin{array}{l}0.0 \\
0.0\end{array}$ & $\begin{array}{l}0.0 \\
0.0\end{array}$ & \\
\hline ALKP (nkat/L) & $\begin{array}{l}\text { Male } \\
\text { Female }\end{array}$ & $\begin{array}{l}1033 \\
483\end{array}$ & $\begin{array}{l} \pm 500 \\
\pm 267\end{array}$ & $\begin{array}{l}1334 \\
317\end{array}$ & $\begin{array}{l}333 \\
283\end{array}$ & $\begin{array}{l}1434 \\
867\end{array}$ & \\
\hline AST (nkat/L) & $\begin{array}{l}\text { Male } \\
\text { Female }\end{array}$ & $\begin{array}{l}233 \\
217\end{array}$ & $\begin{array}{l} \pm 83 \\
\pm 33\end{array}$ & $\begin{array}{l}183 \\
217\end{array}$ & $\begin{array}{l}167 \\
183\end{array}$ & $\begin{array}{l}350 \\
250\end{array}$ & \\
\hline LDH (nkat/L) & $\begin{array}{l}\text { Male } \\
\text { Female }\end{array}$ & $\begin{array}{l}1584 \\
1567\end{array}$ & $\begin{array}{l} \pm 950 \\
\pm 617\end{array}$ & $\begin{array}{l}1100 \\
1484\end{array}$ & $\begin{array}{l}733 \\
867\end{array}$ & $\begin{array}{l}2917 \\
2367\end{array}$ & \\
\hline Creatine kinase (nkat/L) & $\begin{array}{l}\text { Male } \\
\text { Female }\end{array}$ & $\begin{array}{l}7268 \\
8135\end{array}$ & $\begin{array}{l} \pm 3300 \\
\pm 1950\end{array}$ & $\begin{array}{l}5168 \\
7335\end{array}$ & $\begin{array}{l}4701 \\
6251\end{array}$ & $\begin{array}{l}11,919 \\
10,835\end{array}$ & \\
\hline Phosphorus (mmol/L) & $\begin{array}{l}\text { Male } \\
\text { Female }\end{array}$ & $\begin{array}{l}1.2 \\
1.5\end{array}$ & $\begin{array}{l} \pm 0.1 \\
\pm 0.2\end{array}$ & $\begin{array}{l}1.1 \\
1.1\end{array}$ & $\begin{array}{l}1.1 \\
1.0\end{array}$ & $\begin{array}{l}1.4 \\
1.8\end{array}$ & \\
\hline Calcium (mmol/L) & $\begin{array}{l}\text { Male } \\
\text { Female }\end{array}$ & $\begin{array}{l}4.5 \\
4.2\end{array}$ & $\begin{array}{l} \pm 0.2 \\
\pm 0.2\end{array}$ & $\begin{array}{l}4.6 \\
3.5\end{array}$ & $\begin{array}{l}4.3 \\
3.5\end{array}$ & $\begin{array}{l}4.7 \\
4.5\end{array}$ & \\
\hline $\mathrm{Ca}: \mathrm{P}$ ratio & $\begin{array}{l}\text { Male } \\
\text { Female }\end{array}$ & $\begin{array}{l}3.77 \\
2.84\end{array}$ & $\begin{array}{l} \pm 0.35 \\
\pm 0.35\end{array}$ & $\begin{array}{l}3.93 \\
2.69\end{array}$ & $\begin{array}{l}3.28 \\
2.52\end{array}$ & $\begin{array}{l}4.08 \\
3.33\end{array}$ & \\
\hline Glucose (mmol/L) & $\begin{array}{l}\text { Male } \\
\text { Female }\end{array}$ & $\begin{array}{l}3.7 \\
4.5\end{array}$ & $\begin{array}{l} \pm 0.4 \\
\pm 1.8\end{array}$ & $\begin{array}{l}3.6 \\
4.4\end{array}$ & $\begin{array}{l}3.3 \\
2.4\end{array}$ & $\begin{array}{l}4.3 \\
6.8\end{array}$ & \\
\hline Total Protein $(\mathrm{g} / \mathrm{L})$ & $\begin{array}{l}\text { Male } \\
\text { Female }\end{array}$ & $\begin{array}{l}70.0 \\
71.0\end{array}$ & $\begin{array}{l} \pm 1.9 \\
\pm 4.9\end{array}$ & $\begin{array}{l}69.0 \\
70.0\end{array}$ & $\begin{array}{l}69.0 \\
65.0\end{array}$ & $\begin{array}{l}73.0 \\
77.0\end{array}$ & \\
\hline Total Solids (g/L) & $\begin{array}{l}\text { Male } \\
\text { Female }\end{array}$ & $\begin{array}{l}99.0 \\
95.0\end{array}$ & $\begin{array}{l} \pm 3.1 \\
\pm 8.6\end{array}$ & $\begin{array}{l}101.0 \\
95.0\end{array}$ & $\begin{array}{l}95.0 \\
84.0\end{array}$ & $\begin{array}{l}102.0 \\
105.0\end{array}$ & \\
\hline Albumin $(\mathrm{g} / \mathrm{L})$ & $\begin{array}{l}\text { Male } \\
\text { Female }\end{array}$ & $\begin{array}{l}20.0 \\
19.0\end{array}$ & $\begin{array}{l} \pm 1.2 \\
\pm 0.8\end{array}$ & $\begin{array}{l}20.0 \\
19.0\end{array}$ & $\begin{array}{l}19.0 \\
18.0\end{array}$ & $\begin{array}{l}22.0 \\
20.0\end{array}$ & \\
\hline Globulin $(\mathrm{g} / \mathrm{L})$ & $\begin{array}{l}\text { Male } \\
\text { Female }\end{array}$ & $\begin{array}{l}50.0 \\
52.0\end{array}$ & $\begin{array}{l} \pm 0.8 \\
\pm 4.6\end{array}$ & $\begin{array}{l}50.0 \\
50.0\end{array}$ & $\begin{array}{l}49.0 \\
47.0\end{array}$ & $\begin{array}{l}51.0 \\
58.0\end{array}$ & \\
\hline A:G ratio & $\begin{array}{l}\text { Male } \\
\text { Female }\end{array}$ & $\begin{array}{l}0.4 \\
0.5\end{array}$ & $\begin{array}{l} \pm 0.0 \\
\pm 0.1\end{array}$ & $\begin{array}{l}0.4 \\
0.5\end{array}$ & $\begin{array}{l}0.3 \\
0.4\end{array}$ & $\begin{array}{l}0.4 \\
0.6\end{array}$ & \\
\hline Cholesterol (mmol/L) & $\begin{array}{l}\text { Male } \\
\text { Female }\end{array}$ & $\begin{array}{l}18.3 \\
19.0\end{array}$ & $\begin{array}{l} \pm 0.9 \\
\pm 3.6\end{array}$ & $\begin{array}{l}18.1 \\
19.1\end{array}$ & $\begin{array}{l}17.3 \\
14.4\end{array}$ & $\begin{array}{l}19.5 \\
23.3\end{array}$ & \\
\hline Bile acids (mmol/L) & $\begin{array}{l}\text { Male } \\
\text { Female }\end{array}$ & $\begin{array}{l}11 \\
8.3\end{array}$ & $\begin{array}{l} \pm 3.7 \\
\pm 4.5\end{array}$ & $\begin{array}{l}12 \\
8\end{array}$ & $\begin{array}{l}6 \\
3\end{array}$ & $\begin{array}{l}15 \\
14\end{array}$ & \\
\hline Uric acid (mmol/L) & $\begin{array}{l}\text { Male } \\
\text { Female }\end{array}$ & $\begin{array}{l}374.7 \\
719.7\end{array}$ & $\begin{array}{l} \pm 148.7 \\
\pm 345.0\end{array}$ & $\begin{array}{l}273.6 \\
642.4\end{array}$ & $\begin{array}{l}267.7 \\
339.0\end{array}$ & $\begin{array}{l}582.9 \\
1171.8\end{array}$ & \\
\hline
\end{tabular}

A:G ratio, plasma albumin-to-globulin ratio; Ca:P ratio, calcium-to-phosphorus ratio; $A L K P$, alkaline phosphatase; $A S T$, aspartate aminotransferase; $L D H$, lactate dehydrogenase

$* p<0.05$, statistical significance 
can affect interpretation of these analytes. Hematology and biochemistry data are an essential diagnostic tool for the evaluation of the health status in any species as shown by their common use as components of individual or population-level health assessment protocols. Since controlled studies are limited to only some of the most commonly kept reptile species and great variability is common even between individuals belonging to the same genus due to many intrinsic and extrinsic factors (Stacy et al. 2011; Uetz et al. 2018), investigations in various reptile species will contribute to increasing the knowledge on blood data variations. This information can prove useful for various applications, such as medical treatment, physiology, understanding mechanisms of disease, and contributions to population-level conservation efforts.

Intrinsic and extrinsic factors are keystones as a basis for accurate interpretation of clinicopathological data notably in reptiles. The many intrinsic (e.g., species, gender, age, physiological status, breeding season, hibernation) and extrinsic (e.g., analytical methodology, season, temperature, environmental conditions, husbandry, diet, wild vs. captivity) factors can profoundly affect blood cell proportions and influence plasma enzyme activities and protein concentrations (Campbell 2015c). In our study animals, effects of some of the extrinsic and intrinsic factors were kept constant, since all snakes belong to the same zoological institution, are maintained at identical environmental, nutritional, and husbandry conditions, and consistent analytical methodology was applied. In addition to these aforementioned factors that need to be considered in clinicopathological data interpretation, formal reference intervals should be established based on recommendations by the Quality Assurance and Laboratory Standards (QALS) committee of the ASVCP (Friedrichs et al. 2012) using a sample

Fig. 1 Image composite of blood cell types of the Louisiana pine snake (Pituophis ruthveni). a, b Erythroid precursors; $\mathbf{c}$ red blood cells (RBC) stained with New Methylene Blue stain showing mature $\mathrm{RBC}$ with punctate precipitates and two immature stages with abundant reticulum staining; d mitotic figure in immature RBC and polychromatophil; e heterophil; $\mathbf{f}$ heterophil and small lymphocyte; $\mathbf{g}, \mathbf{h}$ azurophil; i thrombocyte clump; j, k monocytes; I basophil. Wright Giemsa stain unless otherwise indicated; $\mathbf{c}, \mathbf{i} \times$ 50 objective; $\mathbf{a}, \mathbf{b}, \mathbf{d}-\mathbf{h}, \mathbf{j}-\mathbf{I} \times 100$ objective

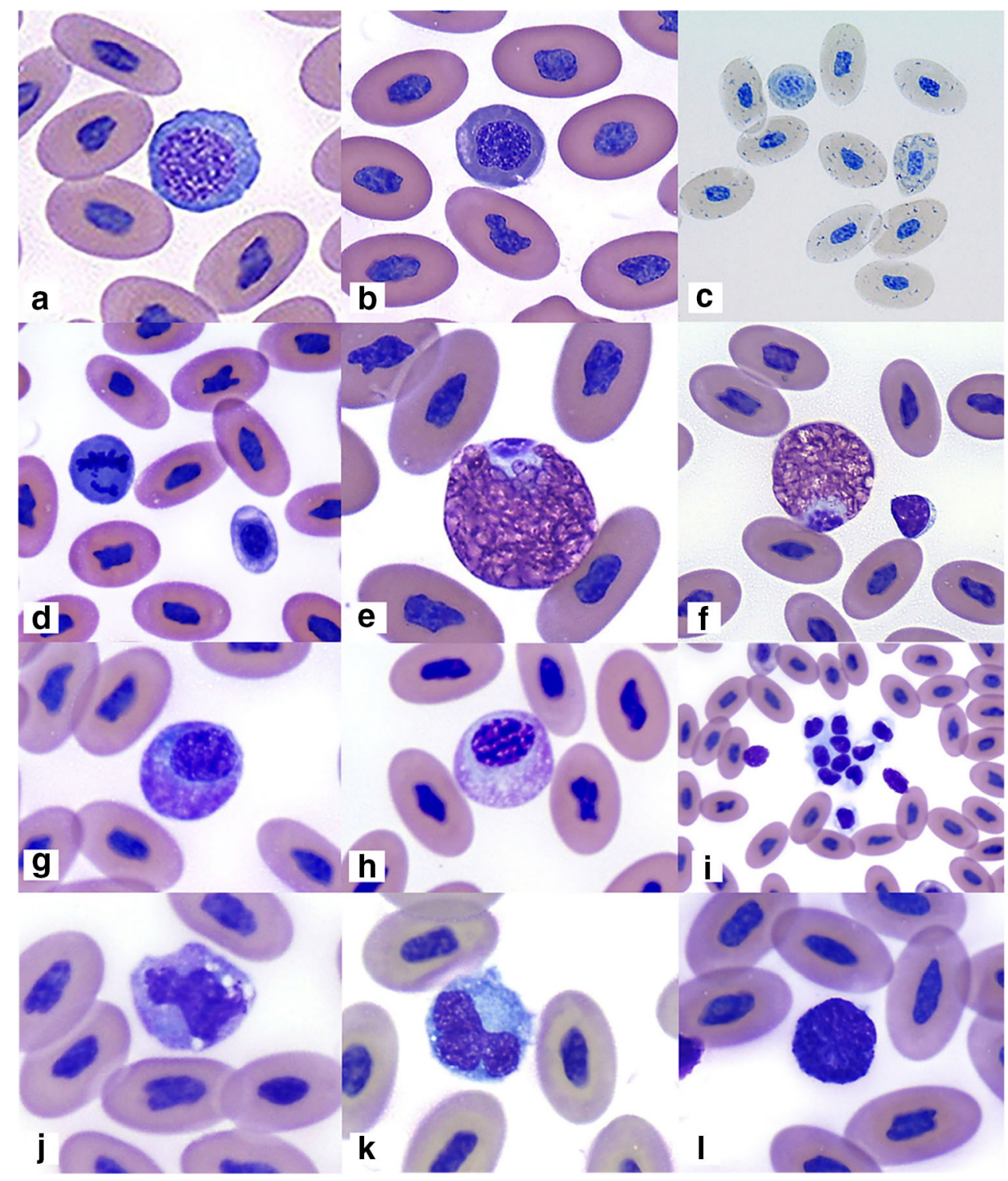


Table 3 Hormone data for apparently healthy, captive Louisiana pine snakes (Pituophis ruthveni) in serum and plasma

\begin{tabular}{|c|c|c|c|c|c|c|}
\hline \multirow{2}{*}{$\begin{array}{l}\text { Age/ } \\
\text { sex }\end{array}$} & \multicolumn{3}{|l|}{ Serum } & \multicolumn{3}{|l|}{ Plasma } \\
\hline & $\begin{array}{l}17 \beta \text {-estradiol } \\
\mathrm{pmol} / \mathrm{L}\end{array}$ & $\begin{array}{l}\text { Progesterone } \\
\mathrm{nmol} / \mathrm{L}\end{array}$ & $\begin{array}{l}\text { Testosterone } \\
\mathrm{nmol} / \mathrm{L}\end{array}$ & $\begin{array}{l}17 \beta \text {-estradiol } \\
\mathrm{pmol} / \mathrm{L}\end{array}$ & $\begin{array}{l}\text { Progesterone } \\
\mathrm{nmol} / \mathrm{L}\end{array}$ & $\begin{array}{l}\text { Testosterone } \\
\mathrm{nmol} / \mathrm{L}\end{array}$ \\
\hline $\mathrm{AF}$ & 1364.47 & 1.02 & $<0.12$ & 956.37 & 0.86 & $<0.12$ \\
\hline $\mathrm{AM}$ & 1030.12 & 2.04 & 35.53 & 711.81 & 1.02 & 40.81 \\
\hline $\mathrm{AF}$ & 1116.39 & $<0.64$ & $<0.12$ & 946.86 & $<0.64$ & $<0.12$ \\
\hline $\mathrm{AM}$ & 1058.28 & $<0.64$ & 8.33 & 810.08 & $<0.64$ & 6.97 \\
\hline $\mathrm{JF}$ & $Q N S$ & $Q N S$ & $Q N S$ & 674.77 & 2.32 & $<0.12$ \\
\hline JF & 1073.91 & 2.19 & $<0.12$ & 800.57 & 1.43 & $<0.12$ \\
\hline $\mathrm{JF}$ & $Q N S$ & $Q N S$ & $Q N S$ & 675.57 & 0.70 & $<0.12$ \\
\hline JF & 948.66 & 0.76 & $<0.12$ & 727.85 & $<0.64$ & $<0.12$ \\
\hline JM & 859.93 & 1.08 & $<0.12$ & 772.53 & $<0.64$ & $<0.12$ \\
\hline
\end{tabular}

$<0.64$ and $<0.12$ are below detection limits for progesterone and testosterone, respectively. Data in italics were excluded from statistical analysis

$Q N S$, quantity not sufficient; $A$, adult; $J$, juvenile; $F$, female; $M$, male set of at least 20 but ideally 120 and appropriate statistical methodology (Cray 2015; Geffre et al. 2009a; Geffre et al. 2009b). This can prove challenging given the often low availability of individuals in a given nondomestic species, which is also a limitation of our study. Despite this limitation, we are using descriptive methodology in order to provide baseline data that represent one step in filling a knowledge gap on hematology, chemistry, and hormone data in this endangered species. Very limited information on hematological data are available for the other 4 species of the same genus Pituophis to date (Dietz and Brodie 1969; Mader et al. 1985).

Hematology data demonstrated life stage and sex differences in LPS. The lower PCV in two juveniles with concurrent evidence of erythroid regeneration indicate regenerative anemia of undetermined inciting cause as these two snakes were otherwise clinically normal. Higher PCV in males compared with females is common in reptile species and consistent with physiological variation (Campbell 2015a; Wilkinson 2004). PCV data of LPS was comparably higher than reported for wild-caught, laboratory-housed gopher snakes (Dietz and Brodie 1969; Mader et al. 1985). The significantly higher lymphocytes in adult compared with juvenile snakes and in adult male versus adult female snakes presumptively present a physiological difference. Such variations of leukocytes by life stage and sex are important to consider in hemogram interpretation. Similar to other snake species, lymphocytes were the predominant WBC type (Divers et al. 1996; Lamirande et al. 1999; Sypek and Borysenko 2009; Troiano et al. 1997). Although azurophils reportedly are often the second most frequent WBC type in snakes (Alleman et al. 1992; Salakij et al. 2002; Troiano et al. 1997), our study animals had very low numbers, which supports the clinical findings of snakes being apparently healthy. In captive-bred cobras, PCV and lymphocytes were higher in wild caught compared with captive-bred snakes, presumably due to nutritional and environmental factors such as exposure to infectious agents and consequent immune stimulation (Salakij et al. 2002). Although our study did not compare captive with wild-caught LPS, this conclusion may be applicable to our captive-bred group of LPS having generally lower WBC.

Plasma biochemical differences in LPS were manifested as life stage but not sex differences and were similar to wildcaught, laboratory-housed gopher snakes (Dietz and Brodie 1969; Mader et al. 1985). Variations of proteins and calcium are most consistent with physiological differences in juvenile and adult LPS. The observed correlation of TS and TP indicates that initial evaluation of TS in in-house laboratories can be useful as an initial TP estimate, keeping the following considerations in mind. As in other species, the refractive index may be affected by hemolysis, lipemia, and other chemical constituents, such as urea and electrolytes (Stockham 2008). The biuret method for TP measurement is considered the gold standard for quantification of TP (Jacobson 1992). For accurate quantification of protein fractions, including albumin and globulins, protein electrophoresis is required (Stockham 2008; Zaias and Cray 2002). The observed higher calcium in adults compared with juveniles presumably resulted from biological variation. Since these samples were collected outside the breeding season, it was anticipated that females and males have similar concentrations of plasma analytes.

There are limited studies on bile acid concentrations in reptiles (Knotek et al. 2009; Knotkova et al. 2008; McBride et al. 2006), with fasting reptiles tending to have values lower than $60 \mu \mathrm{mol} / \mathrm{L}$ (Divers 2000). Even though a significant difference was noted between adults and juveniles in our study, all bile acid data were below $15 \mu \mathrm{mol} / \mathrm{L}$. Further bile acid 
studies in healthy and diseased, fasted and non-fasted snakes, and using appropriate assay validation for reptile species are warranted.

There are some limitations of the endocrine data of our study, given the low number of study animals and data based on a single blood collection in all snakes and considering the complexity of reptile endocrinology and reproduction (Taylor and DeNardo 2011). Studies assessing the relationship between steroid hormones and reproduction in male and female snakes showed wide variations during different stages of reproductive cycles (e.g., vitellogenesis, mating, and gestation) and a wide variability between snake species under various environmental, social, and physiologic influences (Aubret et al. 2002; LeMaster et al. 2001; Pang et al. 1998; Shine 2003; Taylor et al. 2004). Despite the limitations of the present study, our descriptive data represent information obtained in a controlled (temperature, humidity, and feeding habits) group of captive LPS during a non-reproductive season.

The similar hormone data in $17 \beta$-estradiol and progesterone of LPS snakes across animals by life stage and sex outside breeding season was expected. However, the observations of high testosterone concentrations in males and undetectable concentrations in females suggest that testosterone, in addition to visual examination and other manual techniques (i.e., probing), has potential as a non-invasive tool for the assessment of sex status in LPS, warranting further investigation. The significantly lower $17 \beta$-estradiol concentrations in plasma versus serum samples has clinical implications since plasma samples are typically used for reptile blood samples given the higher sample volume yield compared with serum and unpredictability of coagulation which can interfere with chemistry data. Therefore, it is necessary to consider sample type in the interpretation of $17 \beta$-estradiol concentrations.

This study is the first to provide baseline information on hematology, plasma biochemistry, and hormone analytes in endangered LPS. Our data suggests life stage and sex differences that reflect physiological variations and that are necessary to consider in the interpretation of blood analyte data from this species. The data presented herein will be useful for individual animals in managed care, provides an incentive for future investigations, and may have applications for future population-level assessments.

Acknowledgments The authors thank Julie Fields, Jillene Sennon, and Joseph Ramos of the Department of Biomedical and Diagnostic Sciences at the University of Tennessee,

\section{Compliance with ethical standards}

Conflict of interest The authors declare that they have no conflicts of interest.

Ethical approval Zoo Knoxville guidelines for the care and use of animals were followed.
Open Access This article is distributed under the terms of the Creative Commons Attribution 4.0 International License (http:// creativecommons.org/licenses/by/4.0/), which permits unrestricted use, distribution, and reproduction in any medium, provided you give appropriate credit to the original author(s) and the source, provide a link to the Creative Commons license, and indicate if changes were made.

\section{References}

Alleman AR, Jacobson ER, Raskin RE (1992) Morphological and cytochemical characteristics of blood-cells from the desert tortoise (Gopherus-Agassizii). Am J Vet Res 53:1645-1651

Aubret F, Bonnet X, Shine R, Lourdais O (2002) Fat is sexy for females but not males: the influence of body reserves on reproduction in snakes (Vipera aspis). Horm Behav 42:135-147

Campbell TW (2015a) Exotic animal hematology and cytology. Fourth edition. Wiley Blackwell, Ames, 69

Campbell TW (2015b) Hematologic techniques in lower vertebrates in: exotic animal hematology and cytology. Fourth edition. Wiley Blackwell, Ames, pp 199-205

Campbell TW (2015c) Peripheral blood of reptiles in: exotic animal hematology and cytology. Fourth edition Wiley Blackwell, Ames, 6788 , Peripheral blood of reptiles

Cray C (2015) Reference intervals in avian and exotic hematology. Vet Clin North Am Exot Anim Pract 18:105-116

Dietz TH, Brodie ED (1969) Blood ion concentrations as a function of developmental stage in gopher snake, Pituophis Melanoleucus catenifer. Comp Biochem Physiol 30:673-678

Divers SJ (2000) Reptilian liver and gastrointestinal testing. In: Fudge AM (ed) Laboratory medicine: avian and exotic pets. WB Saunders, Philadelphia, pp 205-209

Divers SJ, Redmayne G, Aves EK (1996) Haematological and biochemical values of 10 green iguanas (Iguana iguana). Vet Rec 138:203205

Ealy MJ, Fleet RR, Rudolph DC (2004) Diel activity patterns of the Louisiana pine snake (Pituophis ruthveni) in eastern Texas. Tex $\mathrm{J}$ Sci 56:383-394

Edwards A, Jones SM (2001) Changes in plasma progesterone, estrogen, and testosterone concentrations throughout the reproductive cycle in female viviparous blue-tongued skinks, Tiliqua nigrolutea (Scincidae), in Tasmania. Gen Comp Endocrinol 122:260-269

Friedrichs KR, Harr KE, Freeman KP, Szladovits B, Walton RM, Barnhart KF, Blanco-Chavez J (2012) ASVCP reference interval guidelines: determination of de novo reference intervals in veterinary species and other related topics. Vet Clin Pathol 41:441-453

Geffre A, Braun JP, Trumel C, Concordet D (2009a) Estimation of reference intervals from small samples: an example using canine plasma creatinine. Vet Clin Pathol 38:477-484

Geffre A, Friedrichs K, Harr K, Concordet D, Trumel C, Braun JP (2009b) Reference values: a review. Vet Clin Pathol 38:288-298

Himes JG (2001) Burrowing ecology of the rare and elusive Louisiana pine snake, Pituophis ruthveni (Serpentes: Colubridae). AmphibiaReptilia 22:91-101

Himes JG, Hardy LM, Rudolph DC, Burgdorf SJ (2002) Growth rates and mortality of the Louisiana pine snake (Pituophis ruthveni). J Herpetol 36:683-687

International Union for Conservation of Nature (2019) The IUCN red list of threatened species: Pituophis ruthveni. https://www.iucnredlist. org/species/63874/12723685. Accessed 02 Dec 2018

Jacobson ER (1992) Laboratory investigations. In: Beynon PH, Lawton MPC, Cooper JE (eds) Manual of reptiles. British Small Animal 
Veterinary Association. Iowa State University Press, Ames, pp 5062

Jenkins-Perez J (2008) Hematologic evaluation is mainstay of diagnosis for reptiles. Vet Tech 29:616-624

Jones SM (2011) Hormonal regulation of ovarian function in reptiles. In: Hormones and reproduction of vertebrates, Reptiles, vol 3, pp 89115

Knotek Z, Knotkova Z, Hrda A, Dorrestein GM (2009) Plasma bile acids in reptiles. In: Proceedings of the Association of Reptilian and Amphibian Veterinarians, 16th annual conference, Milwaukee, Wisconsin, USA, 8-15 August, 2009: pp. 124-127

Knotkova Z, Dorrestein GM, Jekl V, Janouskova J, Knotek Z (2008) Fasting and postprandial serum bile acid concentrations in 10 healthy female red-eared terrapins (Trachemys scripta elegans). Vet Rec 163:510-514

Lamirande EW, Bratthauer AD, Fischer DC, Nichols DK (1999) Reference hematologic and plasma chemistry values of brown tree snakes (Boiga irregularis). J Zoo Wildl Med 30:516-520

Lee JW, Devanarayan V, Barrett YC, Weiner R, Allinson J, Fountain S, Keller S, Weinryb I, Green M, Duan L, Rogers JA, Millham R, O’Brien PJ, Sailstad J, Khan M, Ray C, Wagner JA (2006) Fit-forpurpose method development and validation for successful biomarker measurement. Pharm Res 23:312-328

LeMaster MP, Moore IT, Mason RT (2001) Conspecific trailing behaviour of red-sided garter snakes, Thamnophis sirtalis parietalis, in the natural environment. Anim Behav 61:827-833

Mader DR, Horvath CC, Paul-Murphy J (1985) The hematocrit and serum profile of the gopher snake (Pituophis melanoleucas catenifer). J Zoo Anim Med 16:139-140

McBride M, Hernandez-Divers SJ, Koch T, Bush S, Latimer KS, Wilson H, Hernandez-Divers SM, Stedman NL (2006) Preliminary evaluation of pre- and post-prandial 3alpha-hydroxy bile acids in the green iguana, Iguana iguana. J Herpetol Med Surg 16:129-134

Mukaka MM (2012) Statistics corner: a guide to appropriate use of correlation coefficient in medical research. Malawi Med J 24:69-71

Pang SF, Li L, Ayre EA, Pang CS, Lee PPN, Xu RK, Chow PH, Yu ZH, Shiu SYW (1998) Neuroendocrinology of melatonin in reproduction: recent developments. J Chem Neuroanat 14:157-166

Pierce JB, Rudolph DG, Gregory BB, Melder CA (2014) Pituophis ruthveni (Louisiana Pinesnake) growth rates. Herpetol Rev 45(3): 519

Rudolph DC, Burgdorf SJ, Schaefer RR, Conner RN, Maxey RW (2006) Status of Pituophis ruthveni (Louisiana Pine Snake). Southeast Nat 5:463-472

Rudolph DC, Schaefer RR, Burgdorf SJ, Duran M, Conner RN (2007) Pine snake (Pituophis ruthveni and Pituophis melanoleucus lodingi) hibernacula. J Herpetol 41:560-565
Rush EM et al. (2016) Advances in clinical pathology and diagnostic medicine. In: Speer BL (ed) Current therapy in avian medicine and surgery. First edition Elsevier, St. Louis, 461-530

Salakij C, Salakij J, Apibal S, Narkkong NA, Chanhome L, Rochanapat N (2002) Hematology, morphology, cytochemical staining, and ultrastructural characteristics of blood cells in king cobras (Ophiophagus hannah). Vet Clin Pathol 31:116-126

Shine R (2003) Reproductive strategies in snakes. Proceedings of the Royal Society B: Biological Sciences

Stacy NI, Alleman AR, Sayler KA (2011) Diagnostic hematology of reptiles. Clin Lab Med 31:87-108

Stockham SL (2008) Proteins. In: Stockham SL, Scott MA (eds) Fundamentals of veterinary clinical pathology. John Wiley \& Sons, New York, pp 369-413

Sypek J, Borysenko M (2009) Reptiles. In: Rowley AF, Ratcliffe NA (eds) Vertebrate blood cells. Cambridge Univ. Press, Cambridge, pp 211-256

Taylor EN, DeNardo DF (2011) Hormones and reproductive cycles in snakes. In: Hormones and reproduction of vertebrates, Reptiles, vol 3, pp 355-372

Taylor EN, DeNardo DF, Jennings DH (2004) Seasonal steroid hormone levels and their relation to reproduction in the Western diamondbacked rattlesnake, Crotalus atrox (Serpentes: Viperidae). Gen Comp Endocrinol 136:328-337

Troiano JC, Vidal JC, Gould J, Gould E (1997) Haematological reference intervals of the South American rattlesnake (Crotalus durissus terrificus, Laurenti, 1768) in captivity. Comp Haematol Int 7:109 112

Tumkiratiwong P, Meesuk W, Chanhome L, Aowphol A (2012) Reproductive patterns of captive male and female monocled cobra, Naja kaouthia (Lesson, 1831). Zool Stud 51:692-700

Uetz P, Freed P, Hošek J (eds) (2018) The reptile database. http://www. reptile-database.org. Accessed 18 Sep 2018

Vitt LJ, Caldwell JP (2014) Squamates - part II. Snakes. In: Herpetology: an introductory biology of amphibians and reptiles, Fourth edn, pp $597-628$

Wilkinson R (2004) Clinical pathology. In: McArthur S, Wilkinson R, Meyer J (eds) Medicine and surgery of tortoises and turtles. John Wiley \& Sons, pp 141-186

Zaias J, Cray C (2002) Protein electrophoresis: a tool for the reptilian and amphibian practitioner. J Herpetol Med Surg 12:30-32

Publisher's note Springer Nature remains neutral with regard to jurisdictional claims in published maps and institutional affiliations. 